\title{
COMPOSTOS DIAMANTÓIDES NA EXPLORAÇÃO DO PETRÓLEO
}

\author{
F. S. MENDES \\ Universidade Potiguar - UnP \\ franklinmendes@unp.br
}

Artigo submetido em novembro/2014 e aceito em dezembro/2014

DOI: $10.15628 /$ holos.2014.2599

\section{RESUMO}

Os compostos diamantóides se encontram naturalmente no petróleo e se caracterizam pela alta resistência ao craqueamento térmico, à biodegradação e à oxidação. Esses compostos auxiliam na determinação de níveis de craqueamento dos óleos e no reconhecimento de misturas de fluidos hidrocarbônicos provenientes de diferentes pulsos de migração. No presente trabalho foi realizado um levantamento de estudos executados em diversas amostras de óleos de diferentes bacias sedimentares quanto ao seu conteúdo em diamantóides e biomarcadores. As avaliações realizadas nos estudos de caso apresentados permitiram uma melhor avaliação no grau de evolução térmica dos hidrocarbonetos no tocante ao entendimento mais preciso da dinâmica dos processos evolutivos destes compostos em uma bacia sedimentar, o que proporciona uma estimativa mais precisa do risco exploratório e suas implicações técnicas e econômicas, contribuindo com o aumento do conhecimento das bacias petrolíferas.

PALAVRAS-CHAVE: Diamantóides, Evolução Térmica, Reservatórios de Petróleo.

\section{DIAMONDOID COMPOUNDS IN OIL EXPLORATION}

\begin{abstract}
The diamondoid compounds naturally found in petroleum and are characterized by high resistance to thermal cracking, oxidation, and biodegradation. Such compounds help determine levels cracking of oils and recognition of mixtures of hydrocarbon fluids from different pulses of migration. In this paper a survey of studies performed in different oil samples from different sedimentary basins for their content in diamondoid and biomarker was performed. The studies performed in the
\end{abstract}

presented case evaluations have a better assessment of the degree of thermal evolution of hydrocarbons in relation to more accurate understanding of the dynamics of evolutionary processes of these compounds in a sedimentary basin, which provides a more precise estimate of the exploration risk and its implications technical and economic, contributing to the increase of knowledge of petroleum basins.

KEYWORDS: Diamondoid, Thermal Evolution, Oil Reservoirs. 


\section{INTRODUÇÃO}

\subsection{CARACTERÍSTICAS E APLICAÇÕES NA INDÚSTRIA DO PETRÓLEO}

Os diamantóides constituem uma classe de hidrocarbonetos policíclicos saturados que apresentam estrutura molecular semelhante ao diamante. Ocorrem naturalmente no petróleo e têm sido identificados em diversos óleos (Wingert, 1992; Caldas \& Azevedo, 2006).

Devido à sua estrutura molecular, os diamantóides apresentam uma elevada resistência ao craqueamento térmico, à degradação biológica e à oxidação (Lin \& Wilk, 1995). Estes compostos são formados durante ou mesmo após a geração do óleo, pelo rearranjo de hidrocarbonetos policíclicos sob estresse térmico com a presença de um forte ácido de Lewis como catalisador.

O diamantóide mais simples é o adamantano (Figura 1), constituído de uma subunidade estrutural do diamante. Duas subunidades constituem o diamantano, três o triamantano e assim por diante. A série de homólogos dos diamantóides apresenta a fórmula geral $\mathrm{C}_{4 n+6} \mathrm{H}_{4 n+12}$, onde $n$ é um valor inteiro maior ou igual a um (Wingert, 1992).

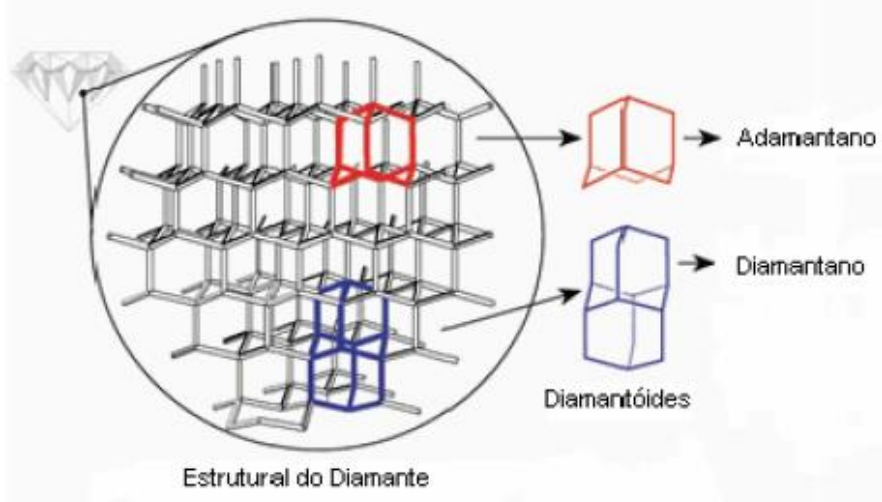

Figura 1 - Estruturas de diamantóides (TAMANQUEIRA, 2006; modificado).

O estudo dos diamantóides tem apresentado aplicações de grande valor no refinamento da caracterização geoquímica de óleos e condensados, auxiliando, por exemplo, na avaliação do grau de evolução térmica de óleos, no reconhecimento de misturas de óleos provenientes de diferentes pulsos de migração, na avaliação do nível de craqueamento secundário (Wingert, op.cit.; Chen et al., 1996; Dahl et al., 1999) e no estudo de óleos leves e condensados, nos quais já não ocorrem biomarcadores. Em suma, o entendimento mais apurado da dinâmica dos processos de geração, migração, acumulação e degradação de hidrocarbonetos em uma bacia sedimentar - e em seu(s) respectivo(s) reservatório(s) - e, desta forma, uma avaliação mais precisa do risco exploratório.

Outra classe de compostos orgânicos complexos utilizados em estudos de reservatórios de petróleo são os biomarcadores. Estes são encontrados em betumes, petróleos e sedimentos, cujas estruturas moleculares apresentam uma relação inequívoca com compostos naturais presentes em organismos vivos, como bactérias, fitoplâncton, zooplâncton e vegetais superiores (Tissot \& Welte, 1984; Peters et al., 2005; Mendes \& Melo Jr., 2005; Mendes, 2006, 2012). O 
termo "fóssil químico" foi usado primeiramente por Eglinton e Calvin (1967) para descrever compostos orgânicos na geosfera cujo esqueleto carbônico sugeriria uma ligação direta com um produto natural conhecido. O termo "biomarcadores", introduzido por Seifert e Moldowan (1981), é atualmente o mais usado.

Estas moléculas representam apenas uma pequena fração do petróleo, porém são de grande interesse para os geoquímicos, pois fornecem informações valiosas sobre os tipos de organismos contribuintes para a matéria orgânica incorporados nos sedimentos, bem como a respeito dos processos que afetam a matéria orgânica ao longo da diagênese e da catagênese (Tissot \& Welte, 1984). A análise de biomarcadores é uma ferramenta rotineira nos estudos do tipo de matéria orgânica, maturação, biodegradação e migração em amostras geológicas (Mackenzie, 1984; Seifert \& Moldowan, 1986; Hunt, 1996; Mendes, 2006).

Dentre os diversos biomarcadores reconhecidos ao longo das últimas décadas, os mais usados nos estudos do petróleo fazem parte dos grupos dos esteranos e dos terpanos (Tissot \& Welte, 1984; Peters \& Moldowan, 1993), cuja estrutura geral é apresentada na Figura 2.

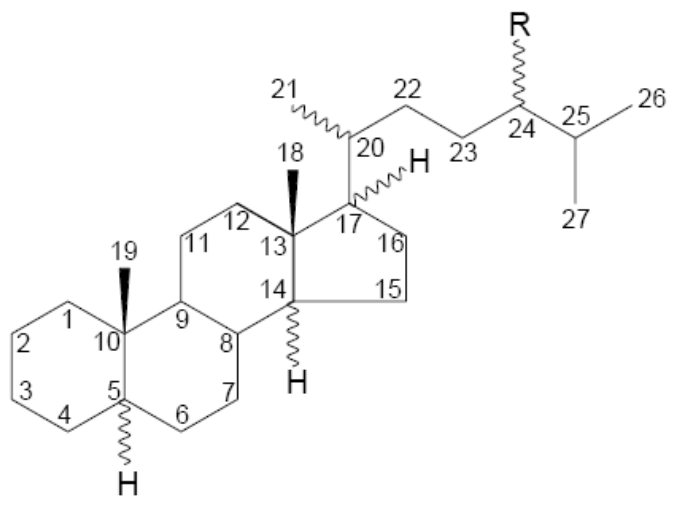

Figura 2 - Estrutura geral de um biomarcador - Esterano (Peters \& Moldowan, 1993).

Diversos estudos avaliativos em reservatórios podem ser realizados utilizando-se compostos biomarcadores e diamantóides. Devido à alta resistência dos diamantóides, eles também podem ser usados na caracterização de óleos submetidos a níveis extremos de biodegradação (Grice et al., 2000). Uma das principais aplicações dos diamantóides consiste na avaliação do grau de maturação de óleos e condensados. Razões de isomerização de esteranos e hopanos têm sido amplamente aplicadas com este fim. Entretanto, a maior parte dessas razões atinge seus valores de equilíbrio antes ou durante a fase compreendida entre o início da "janela de óleo" e o pico de geração de óleo, e, portanto, não pode ser usada na avaliação de óleos com grau de evolução térmica mais elevada (Chen et al.,1996).

No tocante aos estudos de compostos diamantóides, uma questão de interessante para a indústria do petróleo é a deposição dos diamantóides (Figura 3) que pode ser particularmente problemática durante a produção e transporte do gás natural, gás condensado e óleos crus leves, pois, estes compostos, comuns em fluidos de reservatório, podem ocasionar incrustações e seus fenômenos subsequentes (Ex.: tamponamento do fluxo).

A deposição de compostos orgânicos presentes no óleo nas etapas da produção, transporte e processo do petróleo, betumem e outros hidrocarbonetos podem ser significantemente afetados pela floculação e deposição de asfaltenos, resinas, parafinas/graxas, 
diamantóides, organo-metálicos, etc. nas tubulações dos reservatórios, dos poços de óleos, bombas, vasos de depósitos, linhas de transferências e refinaria com consequências econômicas negativas (Ex.: queda de produção, danos aos equipamentos, redução da segurança operacional, etc.). Uma questão de interesse para a indústria de petróleo é quando e quanto dos compostos orgânicos irão flocular sob certas condições de operação (Ex.: Temperatura, pressão, vazão, fluxo). O petróleo cru é normalmente uma mistura de hidrocarbonetos e compostos orgânicos pesados, portanto, se torna necessário avaliar cada um dos constituintes como uma mistura interagindo uma com a outra. O tipo e quantidade do depósito dos compostos orgânicos nos fluidos de petróleo variam dependendo dos hidrocarbonetos presentes no óleo e a percentagem relativa de cada família dos compostos orgânicos.

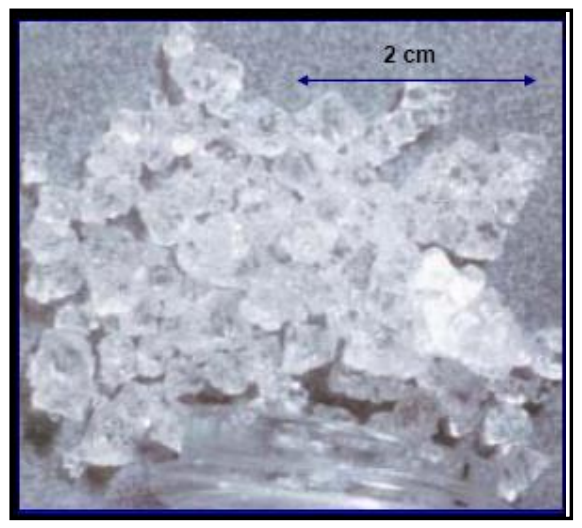

Figura 3 - Precipitado de diamantóides coletado de um campo do Golfo do México (SCHOELL \& CARLSON, 1999).

Apesar de todo o potencial para o refinamento dos estudos de sistemas petrolíferos, poucos têm sido os trabalhos que publicaram resultados de análises de diamantóides em óleos no Brasil até o momento (Dahl et al., 1999; Freitas et al., 2000).

\section{ESTUDO DE CASOS}

Os diamantóides foram primeiramente descobertos e isolados a partir de um petróleo tchecoslovaco em 1933 (Lin \& Wilk, 1995; Dahl et al., 1999). A substância isolada foi chamada de adamantano, cujo nome é proveniente da palavra diamante em grego. A seguir, veremos um levantamento de trabalhos científicos relacionados a estes compostos.

Nos estudos de óleos do sul do Texas, Williams et al. (1986) identificaram alguns adamantanos e observaram que os mesmos não foram afetados pela biodegradação nesta série de óleos, já que, pelos fragmentogramas obtidos, as abundâncias dos íons dos adamantanos foram equivalentes àquelas encontradas em um óleo geneticamente não degradado. Foi sugerida, então, a utilização dos adamantanos em correlações de óleos severamente degradados.

Wingert (1992) fez um estudo mais aprofundado no qual identificou e caracterizou por meio de cromatografia gasosa acoplada a espectrometria de massas, os diamantóides (adamantanos, diamantanos e triamantanos) presentes em óleos do sudoeste do Alabama. O modo de monitoramento seletivo de íons foi usado para intensificar a deteç̧ão dos compostos e 
relatar importantes informações na caracterização dos diamantóides. Dentre elas, cita-se a identificação da série dos triamantanos, até então não reconhecida, e o principal controle do espectro de massas. Esse controle permitiu a identificação do alquil diamantóide pelas seguintes maneiras: substituições dos grupos alquil na estrutura do anel do diamantóide, pelo comprimento do grupo alquil e pelo número de substituições no anel.

Lin \& Wilks (1995) descobriram a existência de um conjunto de polimantanos, incluindo tetramantano, pentamantano e hexamantano em condensados de um reservatório petrolífero localizado na Costa do Golfo dos EUA, a uma profundidade de cerca de $6800 \mathrm{~m}$. Os tetramantanos e pentamantanos foram identificados baseados em seus espectros de massas obtidos usando o modo varredura total. O modo de monitoramento seletivo de íons foi usado para identificar a presença dos compostos do hexamantano. Esta foi a primeira vez que foi reportada a ocorrência natural desses compostos.

Chen et al. (1996) identificaram diamantóides em óleos e rochas geradoras de várias bacias chinesas e aplicaram os resultados para avaliar a maturação térmica de óleos e condensados destas bacias. Alguns aspectos geológicos e geoquímicos relacionados à formação e evolução do petróleo nas bacias puderam ser estudados em detalhe através de dois novos índices de maturação propostos por esses autores; MAl (Methyl Adamantane Index) e MDI (Methyl Diamantane Index); de acordo com as equações (1) e (2).

$$
\begin{aligned}
\mathrm{MAI} & =\frac{\text { 1-Metiladamantane }}{\text { (1-Metiladamantane }+ \text { 2-Metiladamantane })} \\
\mathrm{MDI} & =\frac{\text { 4-Metiladamantane }}{\text { (1-Metiladamantane }+ \text { 3-Metiladamantane + 4-Metiladamantane) }}
\end{aligned}
$$

Com base na correlação entre os dados de reflectância de vitrinita e os índices MDI e MAI medidos em rochas geradoras, Chen et al. (1996) propuseram um esquema de correlação entre as duas razões de diamantóides e o grau de evolução térmica de rochas e óleos. Estes parâmetros combinados com os parâmetros de maturação de hopanos e esteranos puderam ser utilizados para avaliar o nível de craqueamento térmico de óleos e condensados altamente maturos nas bacias estudadas.

Fang et al. (1996) analisaram amostras de gás e condensado na Bacia Yinggehai (China). Com base nos parâmetros de diamantóides propostos por Chen et al. (1996), Fang et al. (1996) concluíram que os condensados e gases analisados foram gerados no início ou dentro da janela de geração de condensado (zona regressiva de geração de óleo).

Dahl et al. (1999) usaram a abundância relativa dos diamantóides, cuja grande estabilidade leva à progressiva concentração ao longo da evolução térmica, para identificar a ocorrência e estimar o avanço do processo de craqueamento do óleo. Estes autores propuseram um método para estimar a maturação térmica de qualquer amostra de hidrocarboneto líquido baseado nas concentrações de estigmastano e diamantóides. Relataram, também, a extensão do craqueamento (conversão de hidrocarbonetos líquidos em gás e pirobetume) com relação às concentrações de diamantóides. Este método é particularmente útil para condensados altamente maduros, onde outros métodos podem não ser confiáveis. 
Os biomarcadores geralmente apresentam ligações que podem ser facilmente rompidas com o aumento da maturação térmica e a sua concentração decresce antes do craqueamento da maioria dos componentes de óleos. Segundo Dahl et al. (1999), diferentes biomarcadores apresentam comportamentos diferentes frente à estabilidade térmica. Os diamantóides, que apresentam estrutura molecular bastante rígida quando comparada a outros componentes do petróleo, tendem a ter concentrações maiores no óleo residual. Desta forma, o craqueamento intenso de um óleo acarreta na destruição da maioria dos componentes e no aumento da concentração de diamantóides.

Para obtenção da correlação entre diamantóides e biomarcadores, Caldas e Azevedo, (2006) sugeriram preferencialmente a utilização da concentração de um dos biomarcadores menos estáveis, o $5 \alpha, 14 \alpha, 17 \alpha(\mathrm{H})$-24-etilcolestano 20R (estigmastano) (Figura 4), pois sua concentração se aproxima de zero no ponto em que a concentração de diamantóides começa a aumentar. A utilização das concentrações dos $3+4$-metildiamantanos foi sugerida pelo fato de que estes compostos, os C15 diamantóides, não são facilmente perdidos por evaporação.

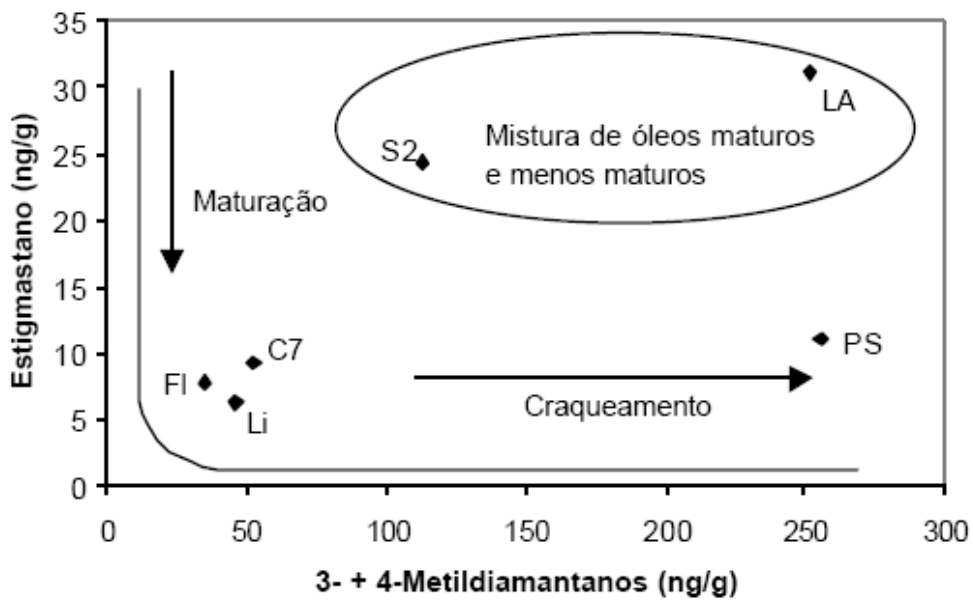

Figura 4 - Curva de correlação das concentrações de diamantóides e biomarcadores em óleos de diferentes maturações térmicas (Caldas \& Azevedo, 2006).

Os estudos de Caldas \& Azevedo (2006 e 2007) se basearam na distribuição de diamantóides e caracterização de biomarcadores em amostras diversas de óleos, inclusive as oriundas da bacia sedimentar colombiana. As análises integradas dos dados de diamantóides e de biomarcadores permitiram reconhecer diferentes graus de evolução térmica entre as referidas amostras de óleos.

As amostras foram fracionadas utilizando cromatografia em coluna de sílica. A fração dos hidrocarbonetos saturados foi eluida com hexano; a dos hidrocarbonetos aromáticos com hexano/diclorometano e os compostos polares (NSO) com mistura diclorometano/metanol.

As frações dos hidrocarbonetos saturados foram analisadas por cromatografia gasosa de alta resolução acoplada a espectrometria de massas (CG/EM) utilizando o monitoramento seletivo de íons. Os íons característicos foram obtidos por análise em varredura linear (SCAN) de padrões adquiridos e por dados da literatura. Foi utilizada uma mistura de parafinas perdeuteradas para a quantificação dos diamantóides e biomarcadores. 
Os resultados foram descritos no gráfico da Figura 4, onde foram correlacionados os dados do biomarcador Estigmastano vs 3+4-Metildiamantanos. Dentre as amostras de óleos estudadas, uma se encontra altamente matura e craqueada, PS; duas indicam ser mistura de óleos maturos craqueados e maturos não craqueados, LA e S2. As outras três amostras se encontram maturas e no início do processo de craqueamento.

Dahl et al. (1999) relataram que cada bacia apresenta uma linha base de diamantóides característica (valores medidos pelas concentrações de 3+4-metildiamantanos) (Figura 5), e que cada uma deve ser determinada individualmente. Os autores citam como exemplo óleos da Formação Monterey (Califórnia) com linha base entre 1 e 2 ppm e óleos da Formação Curuá, na Bacia do Solimões, com linha base na faixa de 2 a 5 ppm.

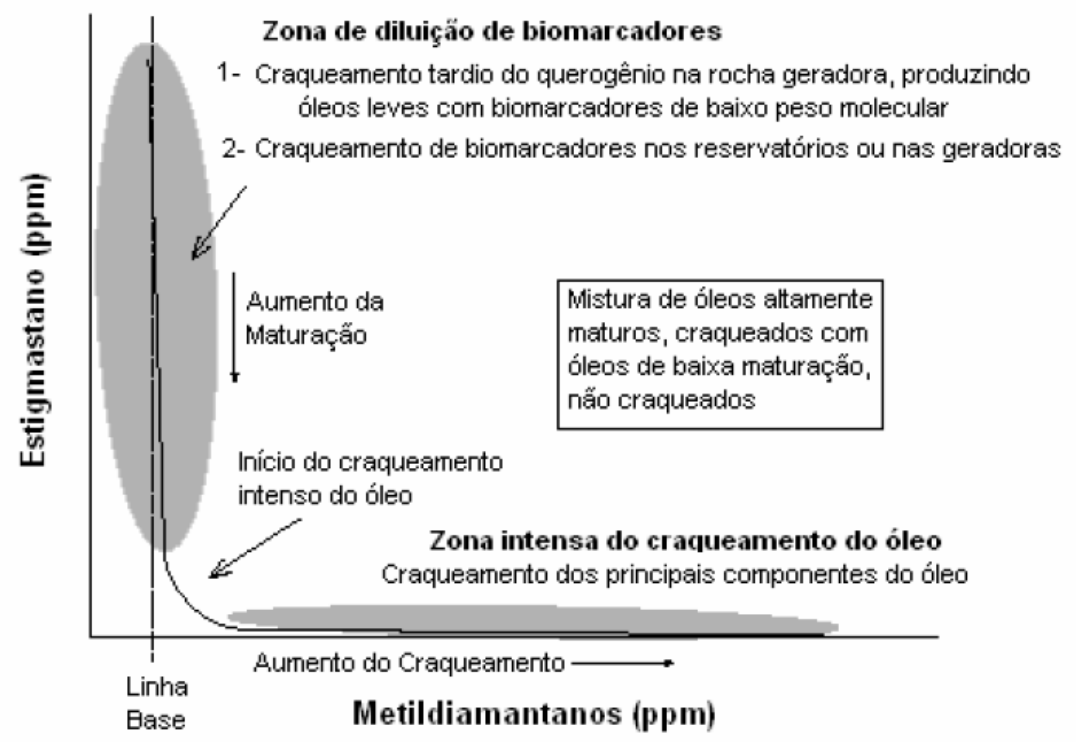

Figura 5 - Correlação entre as concentrações de diamantóides (metildiamantanos) e biomarcadores (estigmastano) em óleos de diferente maturação térmica, craqueado e não craqueado de um sistema específico de petróleo

(DAHL et al., 1999).

Os estudos de Dahl et al. (1999) permitiram também reconhecer misturas de óleos provenientes de diferentes pulsos de migração. A detecção destas misturas de óleos é importante, pois pode resultar em novos conceitos de exploração petrolífera e no melhor entendimento do processo de migração. Óleos que apresentam altas concentrações de diamantóides e de biomarcadores representam misturas de óleos de rochas geradoras de baixa maturação ricas em biomarcadores e rochas geradoras de alta maturação ricas em diamantóides. Esta correlação para avaliar misturas pode ser aplicada a muitas partes do mundo onde óleos podem originar de fontes múltiplas, incluindo em países como o Brasil.

Schoell e Carlson (1999) utilizaram as concentrações de diamantóides como uma medida de destruição do óleo, e determinaram em laboratório que estes compostos podem ser destruídos a uma temperatura de $550^{\circ} \mathrm{C}$. Altas concentrações destes compostos em condensados podem resultar na sua precipitação durante a produção, causando problemas nas linhas de produção.

Freitas et al. (2000) aplicaram os diamantóides no estudo de hidrocarbonetos líquidos gerados e migrados para reservatórios afetados pelo efeito térmico de uma intrusão ígnea 
(soleira de diabásio). As condições térmicas provocadas pela intrusão ígnea foram responsáveis pelo craqueamento de quase toda matéria orgânica existente no intervalo gerador, atingindo diferentes estágios da fase regressiva de geração.

Grice et al. (2000) estudaram o comportamento dos diamantóides em função do grau de alteração de uma série de óleos em duas bacias sedimentares australianas e demonstraram que a distribuição desses compostos é afetada pela biodegradação. Estes autores mostraram o uso dos diamantóides como indicadores de níveis extremos de biodegradação, quando a maioria dos hidrocarbonetos já foi removida, e de mistura de óleos severamente biodegradados e não biodegradados. Estes autores usaram dois parâmetros com a finalidade de avaliar o grau de biodegradação dos óleos em estudo (RAda - Razão dos Adamantanos, Rdia - Razão dos Diamantanos, MA - MetilAdamantano e MD - MetilDiamantano), de acordo com as equações (3) e (4).

$$
\begin{aligned}
& \text { RAda }=\frac{1-\mathrm{MA}+2 \mathrm{MA}}{\mathrm{Ada}} \\
& \mathrm{RDia}=\frac{1 \mathrm{MD}+3 \mathrm{MD}+4 \mathrm{MD}}{\mathrm{Dia}}
\end{aligned}
$$

Schulz et al. (2001) mostraram que os diamantóides além de importantes parâmetros de maturação, também podem ser utilizados com grande eficiência na avaliação das diferentes fácies orgânicas. Estes autores investigaram as distribuições dos diamantóides em extratos provenientes de folhelhos marinhos siliciclásticos, marinhos carbonáticos e carvões de diferentes maturações e introduziram novos parâmetros que, aparentemente, não são afetados pelas mudanças da maturação para suas amostras em particular. Schulz et al. (2001) propuseram parâmetros para distinguir as diferentes fácies orgânicas analisadas: DMDI-1 (índice de dimetildiamantano), DMDI-2 (índice de dimetildiamantano 2) e EAI (índice de etiladamantano), de acordo com as equações (5), (6) e (7).

$$
\begin{gathered}
\text { DMDI-1 }=\frac{3,4 \text {-DiMetildiamantane }}{\text { (3,4-DiMetildiamantane }+(4,9 \text {-DiMetildiamantane })} \\
\text { DMDI-2 }=\frac{4,8 \text {-DiMetildiamantane }}{(4,8 \text {-DiMetildiamantane }+(4,9 \text {-DiMetildiamantane })} \\
\text { EAI }=\frac{\text { 2-EtilAdamantane }}{\text { (2-EtilAdamantane + 1-EtilAdamantane })}
\end{gathered}
$$

Concluíram que as distribuições de dimetildiamantanos e etiladamantanos podem ser usadas para diferenciar matéria orgânica de origem marinha siliciclástica, terrestre e carbonática. Segundo estes autores, os índices DMDI-1 e 2 não são afetados pela maturação permitindo a 
correlação entre óleos não biodegradados e óleos muito biodegradados enquanto o índice EAl é bastante útil dentro da janela de geração de óleo.

Zhang et al. (2005) integraram análises geoquímicas de diferentes frações do óleo e demonstraram que a aplicação dos hidrocarbonetos aromáticos e dos diamantóides apresentam grandes vantagens sobre o uso dos esteranos e terpanos na avaliação do grau de evolução térmica de óleos e condensados na Bacia de Tarim. Estes autores utilizaram vários parâmetros de maturação, tais como razão de dimetilnaftaleno, razão de trimetilnaftaleno, índice de metilfenantreno, razão de metildibenzotiofeno e índices de diamantóides. Zhang et al. (2005) propõem uma escala de correlação entre reflectância de vitrinita e índice de metildiamantano (MDI), cuja equação é dada pela equação (8).

$$
\mathrm{Rz}(\%)=2.4389 \mathrm{x}+0.4364 \mathrm{MDI}
$$

\subsection{Trabalhos Anteriores no Brasil}

Até o momento, pouco foi publicado sobre os diamantóides em bacias sedimentares brasileiras. Em um estudo realizado na Bacia de Solimões, Dahl et al. (1999) relataram um dos exemplos mais claros de aumento na concentração dos diamantóides devido ao craqueamento natural do óleo. Devido ao intenso aquecimento causado pela proximidade a intrusões ígneas, grandes diferenças nas concentrações base de diamantóides, em diferentes partes da bacia, foram constatadas. Óleos não craqueados localizados longe das intrusões, apresentaram concentrações entre 2 e 5 ppm, enquanto óleos recolhidos de reservatórios mais próximos, localizados cerca de $150 \mathrm{~m}$ das intrusões ígneas, se encontraram extensivamente craqueados, com concentrações de metildiamantanos de 32 ppm. Nesta mesma bacia, Freitas et al. (2000) utilizaram os diamantóides como ferramenta auxiliar e mostraram a aplicabilidade nos estudo dos hidrocarbonetos gerados e migrados para reservatórios acima e abaixo de um intervalo gerador afetado pelo efeito térmico de intrusões ígneas (soleiras de diabásio).

Tamanqueira (2006) e Tamanqueira et al. (2005) apresentaram estudos cujo objetivo foi implementar métodos de separação dos diamantóides por cromatografia líquida e de identificação por cromatografia gasosa acoplada à espectrometria de massas (CG-EM) para caracterização dos óleos em bacias sedimentares da margem continental brasileira (Figura 6).

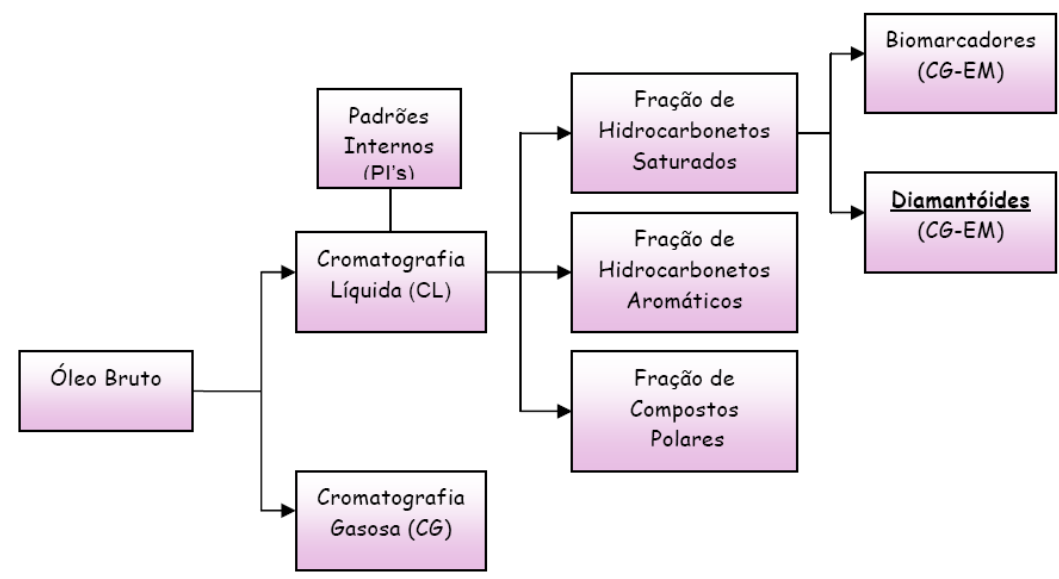

Figura 6 - Fluxograma do procedimento analítico ao qual as amostras de óleo foram submetidas (TAMANQUEIRA et al., 2005 e TAMANQUEIRA, 2006). 
Neste trabalho, foram analisadas amostras de óleo com níveis diferentes de evolução térmica proveniente de cinco bacias sedimentares da margem continental brasileira. $\mathrm{O}$ objetivo principal do trabalho foi detectar e analisar a distribuição dos diamantóides nas amostras selecionadas, aplicando-os como ferramenta de avaliação do grau de evolução térmica. Os diamantóides foram identificados, como pode ser observado na Figura 7, e quantificados através da correlação com padrões internos e padrões autênticos (Tabela 1). Os cromatogramas de massas e os espectros de massas obtidos, também comparados com cromatogramas e espectros de massas publicados, foram utilizados na caracterização dos óleos estudados.

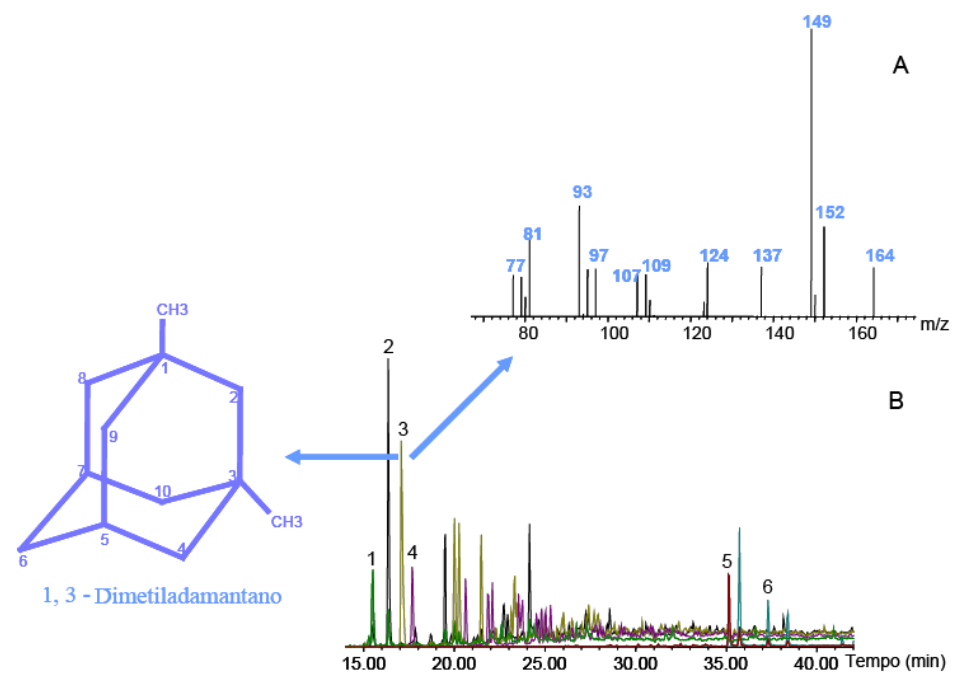

Figura 7 - Análise da fração de hidrocarbonetos saturados de uma amostra de óleo da Bacia A. (A) Espectro de massas: composto 1,3-Dimetiladamantano (pico base o íon $\mathrm{m} / \mathrm{z}$ 149). (B) Cromatograma de massas composto, somatório dos íons $\mathrm{m} / \mathrm{z}$ 135, 136, 149, 163, 187 e 188 (TAMANQUEIRA et al., 2005).

Tabela 1 - Identificação de alguns diamantóides em três bacias brasileiras das cinco bacias estudadas: pico base, tempo de retenção e concentração dos diamantóides selecionados em ng/mg (TAMANQUEIRA et al., 2005).

\begin{tabular}{ccccccc}
\hline Compostos & $\mathbf{N}^{\mathbf{0}}$ Pico & $\begin{array}{c}\text { Pico Base } \\
(\boldsymbol{m} / \boldsymbol{z})\end{array}$ & $\begin{array}{c}\text { Tempo } \\
\text { de } \\
\text { Retenção } \\
(\mathbf{m i n})\end{array}$ & $\begin{array}{c}\text { Bacia A } \\
(\mathbf{p p m})\end{array}$ & $\begin{array}{c}\text { Bacia B } \\
(\mathbf{p p m})\end{array}$ & $\begin{array}{c}\text { Bacia C } \\
\text { (ppm) }\end{array}$ \\
\hline Adamantano & 1 & 136 & 15,6 & 426,3 & 43 & 42,6 \\
1-Metiladamantano & 2 & 135 & 16,4 & 608,9 & 100 & 61,6 \\
1,3-Dimetiladamantano & 3 & 149 & 17,1 & 375,7 & 78,7 & 32,7 \\
2-Metiladamantano & 4 & 135 & 17,7 & 547,5 & 94,1 & 74,8 \\
Diamantano & 5 & 188 & 35 & 140,4 & 13,7 & 8,7 \\
1-Metiladamantano & 6 & 187 & 37,1 & 69,8 & 15,1 & 11,8 \\
\hline
\end{tabular}

Os resultados deste estudo têm corroborado para a importância da aplicação das análises de biomarcadores e diamantóides para a compreensão da dinâmica do processo de migração. A análise integrada de dados de diamantóides com os de biomarcadores permitiu reconhecer os diferentes graus de evolução térmica dos óleos das diferentes bacias brasileiras, já que a 
estabilidade térmica dos diamantóides conduz à concentração progressiva durante o craqueamento do óleo, enquanto a concentração de biomarcadores diminui (Figura 8).

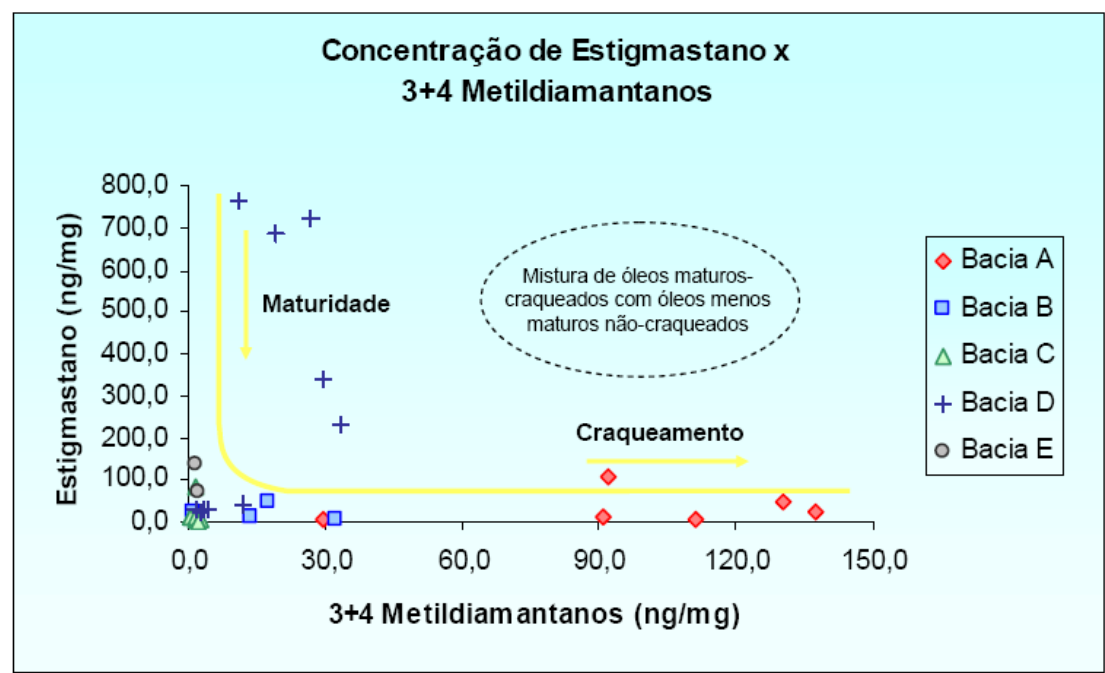

Figura 8 - Correlação entre a concentração de biomarcador (estigmastano) e diamantóides em óleos de diferentes maturidades térmicas, onde a concentração do biomarcadores diminui com o aumento do craqueamento térmico, enquanto a concentração de diamantóides aumenta (TAMANQUEIRA et al., 2005).

Em Jesuino (2005) 19 amostras de óleo de bacias sedimentares da margem continental brasileira foram usadas para aprimorar e disponibilizar um método de separação de diamantóides por cromatografia líquida e de análise, identificação e quantificação por cromatografia gasosa acoplada à espectrometria de massas (CG-EM). Também foram realizadas análises de CG-EM visando à caracterização de biomarcadores nas mesmas amostras. A análise integrada dos dados de diamantóides e de biomarcadores permitiu reconhecer diferentes graus de evolução térmica entre amostras que já haviam alcançado a faixa de equilíbrio da razão de isomerização de esteranos, bem como analisar a possibilidade de misturas de óleos provenientes de pulsos de migração distintos. Parâmetros de diamantóides indicativos da maturação e do tipo de ambiente deposicional propostos na literatura foram correlacionados com razões de biomarcadores com o propósito de testar sua aplicação nas bacias brasileiras.

\section{CONSIDERAÇÕES FINAIS}

Os diversos estudos apresentados neste artigo se utilizaram de métodos analíticos, instrumentais, razões diagnóstico e de gráficos para a obtenção, tratamento e avaliação de dados referentes aos compostos diamantóides e biomarcadores, oriundos de amostras de óleo de uma mesma bacia sedimentar ou de bacias diferentes. A aplicação e o desenvolvimento de métodos analíticos e instrumentais, nos referidos estudos, tiveram por objetivo o refinamento da separação, caracterização e quantificação dos diamantóides em óleos, assim como o reconhecimento das diferentes maturidades e o grau de craqueamento do petróleo.

Nos estudos abordados, foram realizados testes experimentais em análises de diamantóides em óleo. Estes testes se apresentaram mais eficientes quando da ausência da etapa de evaporação, isto é, foram obtidas melhores recuperações destes compostos. Os 
parâmetros biomarcadores se mostraram restritos para a avaliação de óleos com elevado grau de evolução térmica. Tal fenômeno, não apresentou influência nos resultados dos diamantóides, os quais apresentaram dados consistentes.

Razões diagnóstico de Metildiamantano e Metiladamantano podem fornecer indicações da existência de mistura de óleos provenientes de pulsos de migração com níveis diferenciados de maturação em uma bacia petrolífera e seu(s) respectivo(s) reservatório(s). Com a finalidade de avaliar o grau de biodegradação dos óleos, existe a possibilidade de se utilizar outras duas razões: Razão dos Adamantanos e a Razão dos Diamantanos. A utilização do diagrama Estigmastano vs Metildiamantanos permite reconhecer a existência de diferentes graus de evolução térmica do óleo.

As avaliações realizadas nos estudos de caso apresentados, os quais se utilizaram de técnicas de análise quimiométrica, Componentes Principais (PCA) e fatorial, assim como a relação do gráfico Estigmastano vs Diamantóides, permitiram chegar a conclusão de que a análise de diamantóides deve ser incorporada à rotina de um laboratório de geoquímica, pois permite uma melhor avaliação no grau de evolução térmica dos óleos no tocante ao entendimento mais preciso da dinâmica dos processos evolutivos dos hidrocarbonetos em uma bacia sedimentar e no(s) seu(s) reservatório(s), proporcionando uma estimativa mais concisa do risco exploratório e suas implicações técnicas e econômicas, contribuindo com o aumento do conhecimento das bacias petrolíferas e de seus respectivos reservatórios.

\section{REFERÊNCIAS BIBLIOGRÁFICAS}

1. CALDAS, B.; AZEVEDO, D.A. Diamantóides em óleos de bacias sedimentares colombianas. In.: 29a Reunião Anual da Sociedade Brasileira de Química, Brasil, 2006.

2. CALDAS, B.; AZEVEDO, D.A. Aplicação de Diamantóides e Biomarcadores na Avaliação Geoquímica de óleos. In.: XI Encontro da SBQ-Rio de Janeiro Universidade Federal Fluminense, Brasil, 2007.

3. CHEN, J.; Fu, J.; SHEN LIU., D.; ZHANG, J. Diamondoid hydrocarbon ratios: Novel maturity indices for highly mature crude oils. Organic Geochemistry, 25:179-190. 1996.

4. DAHL, J.; MOLDOWAN, M.; PETERS, K.; CLAYPOOLI, G.; ROONEY, M.; MICHAEL, G.; MELLO, M.R.; KOHNEN, M. Diamondoid hydrocarbons as indicators of natural cracking. Nature, 399:54-56, 1999.

5. FANG, H.; SITIAN, L.; YONGCHUAN, S.; QIMING, Z. Characteristics and origin of the gas and condensate in the Yinggehai Basin, offshore South China Sea: evidence for effects of overpressure on petroleum generation and migration. Organic Geochemistry, 24:363-375, 1996.

6. FREITAS, L.C.S.; CERQUEIRA, J.R.; BARBANTI, S.M. Diamondoids as Indicator of Oil Cracking in the Juruá Sub-basin Solimões Basin, Northern Brazil". In 7th Latin-American Congress on Organic Geochemistry (ALAGO), Brasil, p. 33-34, 2000.

7. GRICE, K.; ALEXANDER, R.; KAGI, R. Diamondoid Hydrocarbons as Indicators of Biodegradation in Australian Crude Oils. Organic Geochemistry, 31:67-73, 2000.

8. HUNT, J.M. Petroleum Gechemistry and Geology. $2^{\mathrm{a}}$ Ed., W.H. Freeman and Company, New 
York, 743 p., 1996.

9. JESUINO, L.S. Aplicação dos Diamantóides na Avaliação Geoquímica de Óleos em Bacias Sedimentares Brasileiras. Dissertação de Mestrado. Programa de Pós-Graduação em Engenharia, UFRJ. Rio de Janeiro, RJ. 228p., 2005.

10. LIN, R.; WILK, Z. A. Natural Occurrence of Tetramantane $\left(\mathrm{C}_{22} \mathrm{H}_{28}\right)$, Pentamantane $\left(\mathrm{C}_{26} \mathrm{H}_{32}\right)$, and Hexamantane $\left(\mathrm{C}_{30} \mathrm{H}_{36}\right)$. In.: Deep Petroleum Reservoir", Fuel, 74:1512-1521, 1995.

11. MACKENZIE, A. S. 1984. Application of Biological Markers in Petroleum Geochemistry. In Advances in Petroleum Geochemistry v.1 (Edited by Brooks J. and Welte D.), Academic Press, London, p. 115-215, 1984.

12. MENDES, F.S.; MELO JUNIOR, G. Estudos dos Biomarcadores de Petróleo em Sedimentos de Fundo da Lagoa de Baixo, Polo Industrial de Guamaré - RN / Brasil. Congresso Brasileiro de Geoquímica, 10. Simpósio de Geoquímica dos Paises do Mercosul, 2, Porto de Galinhas / PE, Brasil, CD volume único, Resumo Expandido, cod.. 02-547, 2005.

13. MENDES, F.S. 2006. Estudo Ambiental de Hidrocarbonetos de Petróleo e de Elementos Químicos em Sedimentos de Fundo de um Corpo Lacustre no Município de Guamaré, Estado do Rio Grande do Norte. Tese de Doutorado. Programa de Pós-Graduação em Química, UFRN. Natal, RN. 195 p., 2006.

14. MENDES, F.S. 2012. Biomarcadores de Petróleo no Estudo Ambiental dos Sedimentos de Fundo de um Corpo Lacustre. Revista Eletrônica de Petróleo e Gás - RUnPETRO, Ano 1, $\mathbf{n}^{\mathbf{0}} \mathbf{1}$, P. 6-16., 2012.

15. EGLINTON G.; Calvin, M. Chemical Fossils. Sci. Am., 216: 32-43, 1967.

16. PETERS, K.E.; WALTERS, C.C.; MOLDOWAN, J.M. The Biomarker Guide: Biomarkers and Isotopes in the Petroleum Exploration and Earth History, $2^{\text {nd }}$ Edition, Vol.1 e 2, Cambridge University Press, 2005.

17. PETERS, K.E.; MOLDOWAN, J.M. The Biomarker Guide: Interpreting Molecular Fossils in Petroleum and Ancient Sediments, New Jersey, Prentice-Hall Inc. p. 1-42, 1993

18. RICHARDESON, S.L.; BARUAH, T.; MEHL, M.; PEDERSON, M.R. Theoretical confirmation of the experimental raman spectra of the lower-order diamondoid molecule: cyclohexamantane $\left(\mathrm{C}_{26} \mathrm{H}_{30}\right)$, Chemical Physics Letters, 403: 83-88., 2005.

19. SCHOELL, M. ; CARLSON, R.M.K. Diamondoids and Oil are not Forever. Nature. 399:15-16., 1999.

20. SCHULZ, L.K.; WILHELMS, A., Rein, E., Steen, R.A. Application of Diamondoids to Distinguish Source Rock Facies. Organic Geochemistry, 32:365-375., 2001.

21. SEIFERT, W. K.; MOLDOWAN, J. M. Paleoreconstruction by Biological Markers. Geochimica et Cosmochimica Acta, 45:783-794., 1981.

22. SEIFERT, W. K.; MOLDOWAN, J. M. Use of Biological Markers in Petroleum Exploration. In Biological Markers in the Sedimentary Record (Edited by Johns R. B.), Elsevier, Amsterdam. p.261-290., 1986.

23. TAMANQUEIRA, J.B. Caracterização de Diamantóides em Amostras de Óleo de Bacias Sedimentares Brasileiras. 208p. Dissertação de Mestrado. Programa de Pós-Graduação em Engenharia, UFRJ. Rio de Janeiro, RJ, 2006 
24. TAMANQUEIRA, J.B.; Jesuino, L.S.;Azevedo, D.A.;Gonçalves, F.T.T.; Landau, L. Análise de Diamantóides e sua Aplicação no Estudo de Óleos de Bacias Sedimentares Brasileiras. In: 3o Congresso Brasileiro de P\&D em Petróleo e Gás, 2005. Salvador-BA, 2005.

25. TISSOT, B. P.; WELTE, D.H. Petroleum Formation and Occurrence. 2 ed., New York, SpringerVerlag. - 145 p., 1984.

26. WILLIAMS, J.A.; BJOROY, M.; DOLCATER, D.L.; WINTERS, J.C. Biodegradation in South Texas Eocene oils - Effects on Aromatic and Biomarkers, Organic Geochemistry, 10:451-461., 1986.

27. WINGERT, W.S. G.C.M.S. - Analysis of Diamondoid Hydrocarbons in Smackover Petroleums, Fuel, 71:37-43., 1992.

28. ZHANG, S.; HUANG, G. H.; XIAO, Z.; LIANG, D. Geochemistry of Palaeozoic Marine Petroleum from the Tarim basin, NW China. Part 2: Maturity assessment. Organic Geochemistry, 36:1215-1225., 2005. 